\title{
Emission mechanisms in composite galaxies
}

\author{
M. E. Filho ${ }^{1,2,3}$, F. Fraternali ${ }^{1,4}$, S. Markoff ${ }^{5}$, N. M. Nagar ${ }^{1}$, \\ P. D. Barthel ${ }^{1}$, L. C. $\mathrm{Ho}^{6}$ and F. Yuan ${ }^{7}$ \\ ${ }^{1}$ Kapteyn Institute, P.O. Box 800, 9700 AV Groningen, The Netherlands \\ ${ }^{2}$ IRA, CNR, Via P. Gobetti, 101, 40129 Bologna, Italy \\ ${ }^{3}$ CAUP, Rua das Estrelas, 4150 - 762 Porto, Portugal \\ ${ }^{4}$ ASTRON, P.O. Box 2, 7990 AA Dwingeloo, The Netherlands \\ ${ }^{5}$ MIT, Center for Space Research, 77 Massachusetts Av., R. NE80 - 6035, Cambridge, \\ Massachusetts 02139, USA \\ ${ }^{6}$ OCIW, 813 Santa Barbara Street, Pasadena, California 91101, USA \\ ${ }^{7}$ Purdue University, Department of Physics, 525 Northwestern Av., West Lafayette, Indiana, \\ 47907 - 2036, USA
}

\begin{abstract}
High resolution radio and X-ray observations of a subsample of composite LINER/H II galaxies with arcsec-scale radio cores were obtained. These observations yield a $12 \%$ detection rate of low luminosity AGN among the entire composite sample in the Palomar survey. Four of these low luminosity AGN also possess sub-parsec jet-like radio extensions. While canonical advection-dominated accretion flows may be repsonsible for the radio and X-ray emission in submillijansky cores like NGC 7331, they do not appear consistent with the radio emission in these milliarcsecond-scale radio cores; the latter sources are more likely to have an energetically important contribution from a radio-emitting jet.
\end{abstract}

\section{Sample and Observations}

The subsample of composite galaxies was originally taken from the Palomar sample of bright nearby galaxies (Ho, Filippenko, \& Sargent 1995, 1997a). These sources exhibit both AGN and starburst features in their optical spectra. The composite sources found to possess arcsec-scale (VLA) radio cores were followed-up with VLBA multi-frequency observations and data from the Chandra archive (when available) were obtained. $12 \%$ of all the composite sources in the Palomar sample show evidence for the presence of a low luminosity AGN, i.e., the detection of a hard X-ray and/or compact radio core. Furthermore, four of the sources are found to display milliarcsecond-scale extended emission, reminiscent of a jet structure (Filho et al. 2004).

\section{ADAFs or Jets?}

We wish to use the results of the observations to test models of radio and X-ray emission, namely the canonical accretion flow (ADAF; Yi \& Boughn 1998, 1999) and jet model (Falcke, Körding, \& Markoff 2004; see also Merloni, Heinz, \& di Matteo 2003).

The X-ray data are consistent with the ADAF model for low accretion rates $\left(<10^{-3}\right.$ $\dot{\mathrm{m}}_{\text {edd }}$ ), both for sources with milliarcsecond-scale upper limits and for those with VLBA cores (Fig. 1a). This could suggest the same underlying mechanism for the X-ray production in all sample sources - an ADAF. In fact, in two of the VLBA cores where an X-ray spectral fit was possible (NGC 4552 and NGC 7331), the photon index is consistent with the ADAF predicted value of $\sim 1.4$. 

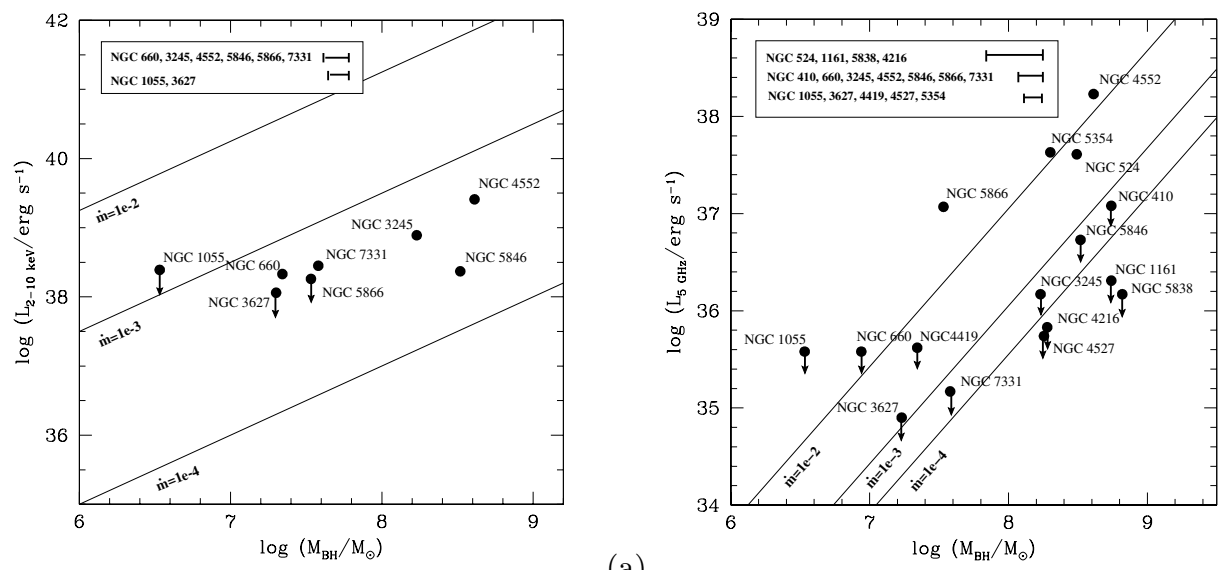

(a)

Figure 1. ADAF models with lines of equi-accretion rate.

Concerning their radio properties, the sources with upper limits to their milliarcsecondscale radio emission are consistent with low mass accretion rates $\left(<10^{-2} \dot{\mathrm{m}}_{\text {edd }}\right)$ in the ADAF scheme (Fig. 1b). In these sources it appears that the accretion flow may simultaneously explain both the nuclear (hypothetical) radio and X-ray emission.

However, the VLBA detected radio cores fall very close to (or above) the ADAF permitted accretion limit $\left(\dot{\mathrm{m}}_{\text {crit }}=10^{-1.6} \dot{\mathrm{m}}_{\text {edd }} ;\right.$ Fig. $\left.1 \mathrm{~b}\right)$. The results suggest that these VLBA cores show more radio emission than can be accounted for from an ADAF. Furthermore, ADAF models predict an inverted radio spectral index while these sources show flat radio spectra. One possible scenario is that these sources possess a compact, radio-emitting jet.

In the jet model, sources with compact inner jets (XRBs, FR I radio galaxies, etc.) lie in a radio/X-ray/black hole mass fundamental plane (Merloni et al. 2003; Falcke et al. 2004). It appears that sources with VLBA cores follow the jet model predicted relation in the sense of excess radio emission. This is entirely consistent with the jet model since these sources are found to possess jet emission on milliarcsecond-scales.

\section{References}

Corbel, S., Novak, M., Fender, R. P., Tzioumus, A. K., \& Markoff, S. 2003, A\&A, 400, 1007

Falcke, H., Körding, E., \& Markoff, S. 2004, A\&A, 414, 895

Filho, M. E., Fraternali, F., Markoff, S., Nagar, N. M., Barthel, P. D., Ho, L. C., \& Yuan, F. 2004, A\&A, in press (astro-ph/0401593)

Ho, L. C., Filippenko, A. V., \& Sargent, W. L. W. 1995, ApJS, 98, 477

Ho, L. C., Filippenko, A. V., \& Sargent, W. L. W. 1997a, ApJS, 112, 315

Markoff, S., Novak, M., Corbel, S., Fender, R., \& Falcke, H. 2003, A\&A, 397, 645

Merloni, A., Heinz, S., \& di Matteo, T. 2003, MNRAS, 345, 1057

Yi, I., \& Boughn, S. P. 1998, ApJ, 499, 198

Yi, I., \& Boughn, S. P. 1999, ApJ, 515, 576 